\title{
Value of Machine Learning with CE-MRI Radiomics for Early Prediction of Pathological Complete Response to Neoadjuvant Therapy in HER2-positive Invasive Breast Cancer
}

\section{Qin Li}

Fudan University Shanghai Cancer Center

\section{Qin Xiao}

Fudan University Shanghai Cancer Center

Jianwei Li

Shanghai Cancer Hospital: Fudan University Shanghai Cancer Center

\section{Zhe Wang}

Human Phenome Institute, Fudan University

He Wang

Human Phenome Institute, Fudan University

Yajia Gu ( $\square$ guyajia@126.com )

Fudan University Pancreatic Cancer Institute Shanghai Pancreatic Institute: Fudan University Shanghai Cancer Center Department of Pancreatic Surgery and Comprehensive Treatment https://orcid.org/00000001-5831-5214

Research article

Keywords: Radiomics, breast cancer, neoadjuvant therapy, machine learning, Magnetic Resonance Imaging

Posted Date: October 26th, 2020

DOl: https://doi.org/10.21203/rs.3.rs-95657/v1

License: (a) (1) This work is licensed under a Creative Commons Attribution 4.0 International License. Read Full License 


\section{Abstract}

Backgrouds To assess the value of radiomics based on multiphases contrast-enhanced magnetic resonance imaging(CE-MRI) for early prediction of pathological complete response(pCR) to neoadjuvant therapy(NAT) in patients with human epithelial growth factor receptor 2(HER2) positive invasive breast cancer.

Methods A total of 127 patients with newly diagnosed primary HER2 positive invasive breast cancer underwent CE-MRI before NAT and performed surgery after NAT. Radiomic features were extracted from the $1^{\text {st }}$ postcontrast CE-MRI phase $\left(\mathrm{CE}_{1}\right)$ and multi-phases $\mathrm{CE}-\mathrm{MRI}\left(\mathrm{CE}_{\mathrm{m}}\right)$. With selected features using a forward stepwise regression, 23 machine learning classifiers based on $\mathrm{CE}_{1}$ and $\mathrm{CE}_{\mathrm{m}}$ were constructed respectively. The performence of classifiers were assessed and compared.

Results For the task of pCR classification, 6 radiomic features from $\mathrm{CE}_{1}$ and 6 from $\mathrm{CE}_{\mathrm{m}}$ were selected for the final machine learning models respectively. The linear SVM based on $C E_{m}$ outperformed the Logistic regression model using $\mathrm{CE}_{1}$ with an $\mathrm{AUC}$ of 0.84 vers 0.69 . In mass and non-mass enhancement groups, the accuracy of linear SVM achieved $76 \%$ and $84 \%$. Whereas in unifocal and unilateral multifocal cases, $79 \%$ and $75 \%$ accuracy were achieved by linear SVM.

Conclusion Multiphases CE-MRI imaging may offer more heterogeneity information in the tumor and provide a non-invasive approach for early prediction of PCR to NAT in patients with HER2 positive invasive breast cancer.

\section{Backgrounds}

HER2 positive breast cancer(non-luminal) is defined as HER2-positive and hormone receptor (HR)negative tumor. It has high malignancy, more recurrence and metastasis, poor prognosis, but is sensitive to neoadjuvant therapy (NAT, Neoadjuvant chemotherapy and anti-HER2 theropy) [1, 2] which is widely used to treat HER2 positive breast cancer[3-5]. NAT could improve the disese-free and overall survival, therefore the pathologic complete response (pCR) to NAT has been proposed as a surrogate endpoint for long-term survival in HER2 positive breast cancer patients[6-9]. Nevertheless, only about $40 \%$ of the patients could achieve pCR after the completion of NAT. Predicting pCR before NAT is desirable for identifying patients who do not benefit from NAT. Identification of pCR can only be conformed by histopathological specimens which is traumatic and does not reflect the heterogeneity of the entire tumor. Thus, a method to predict pCR early and noninvasively remains a major challenge.

Compared with mammography and ultrasound, breast magnetic resonance imaging (MRI) was conformed as the most reliable imaging tool for evaluating the response of tumor to chemotherapy[10, 11]. Many imaging modality of MRI, such as T2WI, DWI and CE-MRI, had been proposed to evaluate the response to NAT in patients with breast cancer[12, 13]. However, CE-MRI is sensitive to perfusion, permeability of tissue microvessels and angiogenic changes and has been identified as the most 
sensitive method for prediction of treatment response to NAT[14-22]. While clinical MRI images of predicting pCR to NAT depend on naked eyes, radiomics can translate medical images into highdimensional datas which reflect not only macroscopic but also the cellular and molecular properties of tissues $[23,24]$. Radiomics is the bridge to combine medical imaging and personalized medicine. The purpose of radiomic analysis is to generate image-driven biomarkers which can provide a deeper understanding of microenvironment and spatial heterogeneity in tumor noninvasively.

To date, previous studies have indicated that radimics model can detect the pCR to NAT in breast cancer $[13,14,21,25]$. However, there are no studies published that specifically assess the performance of MRI radiomics in detecting pCR to NAT in HRE2 positive subtype. In addition, these studies demonstrated the feasibility and potential benefits of radiomics in $\mathrm{PCR}$ prediction in breast cancer only using $\mathrm{CE}_{1}$ which only reflects the spatial heterogeneity of the tumor. Nevertheless, features derived from multi-phases of contrast enhanced MRI images can imply much information changing over time points[26].

In the present study, the MRI scan and treatment protocol for all patients were consistent. We unilized all phases of contrast enhanced MRI images calculating new sequential texture features changing over time points and compared the predictive ability of features from $C_{1}$ and $C E_{m}$. We aimed to construct a radiomics model for personalized pCR evaluation after NAT in HER2 positive invasive breast cancer.

\section{Materials And Methods}

\section{Patient Population}

This retrospective study was approved by our institutional review board, which waived informed consent. In total, 127 female patients who underwent conserving breast surgery or radical mastectomy between January 2012 and December 2018 were consecutively enrolled in the study according to the inclusion and exclusion criteria. The flowchart of the study is shown in Fig. 1.

Inclusion criteria were as follows: (1) patients had primary invasive HER2-positive breast cancers ( $\geq$ T2, and/or positive nodal status) confirmed by biopsy; (2) patients received complete standard treatment(4 6 cycles of $\mathrm{PCH}$ ) with no prior treatment before NAT; (3) pretreatment MRI data obtained using the 1.5-T MR scanner (Aurora Dedicated Breast MRI System; USA; Aurora); (4) the course of NAT need to be monitored with $\mathrm{MRI}$; (5) underwent modified radical mastectomy or breast conservation within one month after completion of NAT; (6) available clinicopathologic data (age, menopausal status, tumor size, Ki-67 index, MRI-reported T stage, N stage, surgery type, FGT, BPE, numbers of lesion, enhancement pattern).

Exclusion criteria were as follows: (1) Metastasis elsewhere in the body; (2) not complete standard treatment; (3) cancelled surgery or not undergo surgy at our hoapital; (4) poor MR image quality resulting from poor contrast injection or motion artefacts; (5) Occult breast cancer.

\section{Magnetic Resonance Imaging and Imaging Analysis}


For all patients, axial breast CE-MR were performed before NAT on a 1.5-T MR scan (Aurora Dedicated Breast MRI System; USA; Aurora) using a breast unique transmit/receive coil in the prone position. The CE-MRI protocol included: (a) T1-weighted fat-saturated precontrast sequence, (b) multiple (3 phases) T1weighted fat-saturated postcontrast sequences that were acquired after intravenous administration of a $0.1 \mathrm{mmol} / \mathrm{kg}$ dose of Gd-DTPA (Magnevist; Bayer-Schering Pharma, Berlin, Germany). Scan parameters were TR/TE = 29.0/4.8 ms; FOV = $36 \mathrm{~cm} \times 36 \mathrm{~cm}$; matrix = $360 \times 360 ; 108$ slices; slice thickness = $1.5 \mathrm{~mm}$. The scanning time of each phase was about 3 minutes, and the number of single-phase scanning layers was 108.

\section{Treatment regimen and criteria for $\mathrm{PCR}$}

All patients received $4 \sim 6$ cycles of $\mathrm{PCH}$ regimen (paclitaxel + carboplatin + trastuzumab; paclitaxel: $80 \mathrm{mg} / \mathrm{m}^{2}$, carboplatin: $A U C=2$, herceptin was initially measured at $4 \mathrm{mg} / \mathrm{kg}$ and maintained at $2 \mathrm{mg} / \mathrm{kg}$; Once a week, 3 times is a cycle of treatment) and then underwent radical mastectomy or breast conservation.

Pathological response was determined by pathologist. pCR was defined as the absence of residual invasive tumor cells in the breast and axillary lymph nodes (ypTO/is + ypNO), as determined microscopically, in surgical specimens.

\section{Tumor Masking and Inter-Observer Reproducibility Evaluation}

When drawing the outline of the tumor, we obey the following principles. First, regardless of whether the lesion presented mass or non-mass enhancement, tumour regions of interest (ROI) were delineated along the contour of the tumor on the 1st postcontrast CE-MRI scan (the peak enhanced phase of the multiphase CE-MRI where the border of leision was the most obvious). Second, the ROls of breast cancer were drawn manually on each slice including the necrosis and blood areas to get the 3D segmentation of the whole tumor. Last, if the tumor is unilateral multifocal lesion, the largest one will be selected as the object. And then the 3D segmented contouring based on the 1st postcontrast images were propagated to pre-contrast and the other two post-contrast phases of CE images. The segmentation of whole tumour was performed on the 3D-Slicer software(version 4.10.2, https://www.slicer.org).

Inter-observer reproducibility of ROI detection and radiomic feature extraction were measured using $\mathrm{CE}_{1}$ of 30 randomly chosen samples. Two experienced radiologists(QL and QX) performed the ROI delineation independently, and then radiomic features extracted from the above two ROls were compared to get the inter-class correlation coefficient. An ICC score greater than 0.8 was interpreted to reach satisfactory agreement. ICC for radiomic features was defined as high (ICC $\geq 0.8)$, medium(0.8 > ICC $\geq 0.5)$, or low $(\mathrm{ICC}<0.5)$.

\section{Feature extraction}


Radiomic shape and texture features were calculated with PyRadiomics package(https://pyradiomics.readthedocs.io/en/latest/index.html) in the Python software (v. 3.7, Python Software Foundation, https://www.python.org/). In total, three groups of imaging features(Supplementary material 1) for each patient were extracted from normalized pretreatment CE-MRI scans: Group 1( $\left.\mathrm{CE}_{\text {shape }}\right)$ consisted of 14 shape-based features derived from the 1st postcontrast $\mathrm{CE}$ images; Group 2( $\left.\mathrm{CE}_{\text {texture }}\right)$ comprised 93 texture features based on each phase $\mathrm{CE}-\mathrm{MRI}$ independently, yielding 372 features; Group $3\left(\mathrm{CE}_{\text {sequential }}\right)$ comprised of 930 sequential features, which were caculated from 93 texture features to characterize the textural changes over time points. In group 3, ten new sequential features from each texture feature were caculated (Algorithms for the ten new sequential features were shown in Supplementary material 2). The first six features, including mean, variance, kurtosis, skewness, energy and entropy, were extracted from each individual subject. The other four features, including Kendall-tau-b, conservation, stability, and dispersion, were calculated from the interactive information between the current subject and the remainder of the subjects. All these features have been used in previous radiomic studies[26].

\section{Feature selection method and machine learning-based classification construction}

To avoid severe multicollinearity or overfitting, a forward stepwise regression was used to select potential important features(stepwisefit function in Matlab 2016, The MathWorks, Natick, MA). And then, the number of selected features was determined by AIC(Akaike information criterion).

With useful features, radiomic machine learning models based on the 1st postcontrast CE-MRI scan individually and multi-phases CE-MRI scans were constructed respectively. Twenty-three classifiers were constructed to discriminate whether a patient achieved PCR: including three decision tree classifiers(fine tree, medium tree, and coarse tree), two discriminant analysis classifiers(Linear Discriminant Analysis LDA and quadratic discriminant analysis QDA), six SVM classifiers(linear, quadratic, cubic, fine gaussian, medium gaussian, and coarse gaussian), four k nearest neighbor (KNN) classifiers(fine KNN, medium $\mathrm{KNN}$, coarse KNN, and weighted KNN), and five ensemble learning classifiers(subspace discriminant, subspace KNN, the AdaBoost algorithm with decision tree, the bootstrap-aggregated (bagged) tree algorithm with decision tree, and the RUSBoost algorithm with decision tree). All the models were conducted using the 5 -fold cross validation, whereby $80 \%$ of cases as training set while $20 \%$ as testing set. The procedure was repeated for ten rounds to average the estimates of performance.

The feature selection and machine learning classification were achieved using the Statistics and Machine Learning Toolbox in MatLab (v. R2018a; The MathWorks, Natick, MA).

\section{Performence of optical model in different types of carcinoma}

The performence of the machine learning-based classification were assessed by their accuracy, sensitivity, specificity, and AUC. The optical machine learning classification was used to discriminate pCR vs. non-pCR in mass and non-mass groups, uni-focal and unilateral multi-focal groups respectively. The AUC values was calculated to assess the performence of the model. 


\section{Radiomics signature building}

The Rad-score of each patient was calculated via a linear combination of potential features, weighted by their respective coefficients. The formula of Rad-score were shown in Supplementary material 3 and4.

\section{Statistical analysis}

Descriptive statistics were summarized as mean \pm standard deviation (SD). Comparisons between $\mathrm{pCR}$ and non-pCR groups were made with the $t$ test or Mann-Whitney $U$ test for quantitative variables and with the $X^{2}$ test or Fisher's test for qualitative variables. All statistical analysis and statistical drawing were performed by MATLAB 2018a software and python3.6. p less than 0.05 was considered statistically significant.

\section{Results}

\section{Clinical characteristics}

A total of 127 lesions from 127 women (mean age, 51.2 years; age range, 24-84 years) were ultimately evaluated. Fifty-four of 127 patients(42.52\%) achieved PCR in the study. Among the 127 cases of HER2 positive breast cancer, 89 cases presented mass enhancement and 38 cases presented non-mass enhancement, while 96 patients were with uni-focal cancers and 31 patients had unilateral multi-focal lesions. There were no significant difference in clinical features and MRI morphology between $\mathrm{PCR}$ and non-pCR groups(shown in Table 1).

ICC

ICC for radiomic features was from 0.9522 to 0.9762 between the two radiologists QL and QX.

\section{Selected features}

For the $\mathrm{CE}_{1}$ and $\mathrm{CE}_{m}, 6$ features from $\mathrm{CE}_{\text {texture }}$ and 6 features from $C \mathrm{E}_{\text {sequential }}$ were selected for the final machine learning models respectively. The potential features were shown in Supplementary material 5 and 6.

\section{Performance of the machine learning-based classification}

With the selected six features from $\mathrm{CE}_{1}$, the accuracy of 23 classifiers ranged from $57.5-68.5 \%$ (Table 2). The optimal model was Logistic regression yielding the highest accuracy of $68.5 \%$, with an AUC of 0.69 .

With the selected six features from $\mathrm{CE}_{m}$, the accuracy of 23 classifiers ranged from $40.9-79.5 \%$ (Table 3). The best accuracy was achieved based the on linear support vector machine (SVM) in conjunction with 6 sequential features based on CE time points, with an AUC of 0.84 . 
The linear SVM based on $\mathrm{CE}_{\mathrm{m}}$ showed an improved performance, with an AUC of 0.84(Fig. 2) over Logistic regression using $\mathrm{CE}_{1}(\mathrm{AUC}=0.69$, Fig. 3).

In mass and non-mass enhancement groups, the accuracy of linear SVM achieved $76 \%$ and $84 \%$. Whereas in uni-focal and unilateral multi-focal cases, the accuracy of linear SVM achieved $79 \%$ and $75 \%$.

\section{Rad-score building}

The Rad-score caculated from the two optical models were significantly higher in the pCR group compared to the non-pCR group (Fig. 4). However, the liner SVM based on multiphase CE-MRI outperformed the Logistic Regression using the single 1st post-contrast CE-MRI sequence according to the boxplot.

\section{Discussion}

In the study, we investigated the ability of machine learning models based on pretreatment CE-MRI to predict pCR to NAT in patients with HER2 positive invasive breast cancer. Sequential texture features changing over time points of pretreatment CE-MRI were proposed with better performence compared with traditional texture features based on the single 1 st phase of postcontrast T1-weighted imaging at CE-MRI imaging. The outperformance of sequential texture features indicated that combining multiphases of pretreatment CE-MRI could be helpful for the early prediction of $\mathrm{pCR}$ to NAT in HER2 positive invasive breast cancer.

In order to predict response to chemotherapy in patients with breast cancer, previous studies[13-15] utilize texture measures extracted from $\mathrm{CE}_{1}$. Herein, our study made full use of all phases of CE-MRI images and compared the prediction ability of machine learning models based on $\mathrm{CE}_{1}$ and $\mathrm{CE}_{\mathrm{m}}$. For the 1 st phase, the 6 radiomic features selected for the final models comprised 2 features from first order, 1 from GLCM, 2 from GLSZM and 1 from GLRLM. The potential model based on $\mathrm{CE}_{1}$ yielded the highest accuracy of $68.5 \%$ with an AUC of 0.69 , which was similar to the results of recent reports in HER2 positive subgroups[13-15]. For multiphases, all the selected 6 radiomic features were obtained from $\mathrm{CE}_{\text {sequential, }}$ comprising 2 features caculated frrom kurtosis, 2 from entropy, 1 from dispersion and 1 from stability. Amoung them, kurtosis and entropy had been identified as biomarkers of response to neoadjuvant chemotherapy in previous reviews[13][27]. The optical model was able to perform robustly. Specifically, the AUC of the final model was 0.84 , which was significantly higher than that of previous reports[12, 25] based on the 1st phase. The result revealed that sequential texture features captured more detailed information of tumor complexity and heterogeneity that indistinguishable to the 1 st phase. Besides, the sequential texture features changing over time points had been identified to be useful in the subtype classification of breast cancer[26]. Specially, the optical model linear SVM performed well in predicting pCR to NAT in HER2 positive patients stratified by breast cancer enhancement pattern and number. In mass and non-mass enhancement groups, the accuracy achieved $76 \%$ and $84 \%$. Whereas in uni-focal and unilateral multi-focal cases, the accuracy were $79 \%$ and $75 \%$ respectively. While in many studies[12, 
$14,24,25]$, non-mass enhancement and unilateral multifocal cases were excluded, our model performed well without regarding to tumor morphology and number. Several studies suggested that non-mass enhancement and multifocal or multicentric[28-30] tumors were more frequently present in HER2positive subtype. For patients with non-mass enhancement and multifocal breast carcinoma, neoadjuvant therapy was more recommended to downstage and improve prognosis[31, 32]. In our study, the rates of non-mass enhancement and multifocal lesions were $28.35 \%(38 / 127)$ and $25.20 \%(32 / 127)$, respectively. So it is more scientific to include non-mass enhancement and multifocal cases in the study of predicting the response to NAT in HER2 positive breast cancer.

In our research, all patients underwent MRI examination with the same scanner and treated with the consistent regimens of 4-6 cycles of PCH. The linear support vector machine (SVM) was the optimal model with an AUC of 0.84 . The prediction performence was greater than a recent study[12] on HER2 positive subgroup in which the corresponding AUCs was 0.70. A possible explanation is that the treatment reagions for training set and validation are not uniform. Another study[25] combining the HER2 positive and TN breast cancers as one subgroup concluded that the AUCs of model of predicting pCR to NAC were less than 0.80 not only in the training sets but also in the validation sets. Nevertheless, analysis suggested that there was a difference in $\mathrm{pCR}$ rates after NAC for HER2-positive and triple-negative breast cancers. To the end, a study [14]concluded that a model using intratumoral and peritumoral radiomics was able to predict pCR with an AUC of 0.80 in HER2 positive breast cancers which was similar to our result. Previous studies [33-35] have shown that radiological characteristics may be affected by differences in MRI magnetic intensity. So the same scanner and uniform treatment regions can enhance robustness of the omics and were benificial to the establishment of a better prediction model.

Rad-score were calculated for each patient with equation of the optical model. Our results indicated that the Rad-score in the pCR group was significantly higher compared to the non-pCR group. This was successfully conformed in treatment evaluation in patients with locally advanced rectal cancer[36].

The work presented in our study had several limitations. First, this was a retrospective study and all patients were recruited from a single center. Second, we compared the performence of machion learning models based on radiomic of pretreatment the 1st postcontrast sequence and multiphases CE-MRI imaging. However, the study lacked an independent validation cohorts, external validation of our results pending adequate patient follow-up will be entailed in the future work.

\section{Conclusion}

Multiphases CE-MRI imaging may offer more heterogeneity information in the tumor and provide a noninvasive approach for early prediction of $p C R$ to NAC in patients with HER2 positive invasive breast cancer.

\section{Abbreviations}


AUC Area under the receiver operating characteristic curve

CE Contrast enhanced

NAT Neoadjuvant therapy

GLCM Gray-level co-occurrence matrix

GLRLM Gray-level run length matrix

GLSZM Gray-level size zone matrix

GLDM Gray level dependence matrix

ICC Intraclass correlation coefficient

ROC Receiver operating characteristic curve

ROI Region of interest SVM Support vector machine

PCR Pathological complete response

RUS Random undersampling

\section{Declarations}

\section{Ethics approval and consent to participate}

Written informed consents were waived due to the study design. The medical ethics committee of Fudan university cancer hospital approved this retrospective study and confirmed that the data was anonymized and maintained with confidentiality.

\section{Consent for publication}

Not applicable.

\section{Availability of data and materials}

All data generated or analysed during this study are included in this published article and its supplementary information files.

\section{Competing interests}

The authors declare that they have no competing interests.

\section{Funding}


This study has received funding by the Application of Health Education in Breast Cancer Screening (No. 2016YFC1303003).

Authors' contributions

Conception and design: LQ and XQ. Acquisition of data: LQ,XQ, LJW. Analysis and interpretation of data: $L Q, X Q, W Z$ and WH. Writing, review, and revision of the manuscript: LQ, XQ, WZ, WH, LJW and GYJ. Study supervision: GYJ and WH. All authors have read and approved the manuscript.

Acknowledgements

Not applicable.

\section{References}

1. Perez EA, Suman VJ, Davidson NE, Martino S, Kaufman PA, Lingle WL, Flynn PJ, Ingle JN, Visscher D, Jenkins RB: HER2 testing by local, central, and reference laboratories in specimens from the North Central Cancer Treatment Group N9831 intergroup adjuvant trial. J CLIN ONCOL 2006, 24(19):30323038.

2. Lee HJ, Park IA, Park SY, Seo AN, Lim B, Chai Y, Song IH, Kim NE, Kim JY, Yu JH et al: Two histopathologically different diseases: hormone receptor-positive and hormone receptor-negative tumors in HER2-positive breast cancer. Breast Cancer Res Treat 2014, 145(3):615-623.

3. Steenbruggen TG, van Ramshorst MS, Kok M, Linn SC, Smorenburg CH, Sonke GS: Neoadjuvant Therapy for Breast Cancer: Established Concepts and Emerging Strategies. DRUGS 2017, 77(12):1313-1336.

4. von Minckwitz G, Untch M, Blohmer JU, Costa SD, Eidtmann H, Fasching PA, Gerber B, Eiermann W, Hilfrich $\mathrm{J}$, Huober $\mathrm{J}$ et al: Definition and impact of pathologic complete response on prognosis after neoadjuvant chemotherapy in various intrinsic breast cancer subtypes. J CLIN ONCOL 2012, 30(15):1796-1804.

5. Cortazar P, Zhang L, Untch M, Mehta K, Costantino JP, Wolmark N, Bonnefoi H, Cameron D, Gianni L, Valagussa $\mathrm{P}$ et al: Pathological complete response and long-term clinical benefit in breast cancer: the CTNeoBC pooled analysis. LANCET 2014, 384(9938):164-172.

6. Spring L, Greenup R, Niemierko A, Schapira L, Haddad S, Jimenez R, Coopey S, Taghian A, Hughes KS, Isakoff SJ et al: Pathologic Complete Response After Neoadjuvant Chemotherapy and LongTerm Outcomes Among Young Women With Breast Cancer. J Natl Compr Canc Netw 2017, 15(10):1216-1223.

7. Slamon D, Eiermann W, Robert N, Pienkowski T, Martin M, Press M, Mackey J, Glaspy J, Chan A, Pawlicki M et al: Adjuvant trastuzumab in HER2-positive breast cancer. N Engl J Med 2011, 365(14):1273-1283. 
8. Perez EA, Romond EH, Suman VJ, Jeong JH, Sledge G, Geyer CJ, Martino S, Rastogi P, Gralow J, Swain SM et al: Trastuzumab plus adjuvant chemotherapy for human epidermal growth factor receptor 2-positive breast cancer: planned joint analysis of overall survival from NSABP B-31 and NCCTG N9831. J CLIN ONCOL 2014, 32(33):3744-3752.

9. Alberro JA BBDP: Long-term outcomes for neoadjuvant versus adjuvant chemotherapy in early breast cancer: meta-analysis of individual patient data from ten randomised trials. $\angle A N C E T O N C O L$ 2018, 19(1):27-39.

10. Yeh E, Slanetz P, Kopans DB, Rafferty E, Georgian-Smith D, Moy L, Halpern E, Moore R, Kuter I, Taghian A: Prospective comparison of mammography, sonography, and MRI in patients undergoing neoadjuvant chemotherapy for palpable breast cancer. AJR Am J Roentgeno/ 2005, 184(3):868-877.

11. Rosen EL, Blackwell KL, Baker JA, Soo MS, Bentley RC, Yu D, Samulski TV, Dewhirst MW: Accuracy of MRI in the detection of residual breast cancer after neoadjuvant chemotherapy. AJR Am J Roentgenol 2003, 181(5):1275-1282.

12. Liu Z, Li Z, Qu J, Zhang R, Zhou X, Li L, Sun K, Tang Z, Jiang H, Li H et al: Radiomics of Multiparametric MRI for Pretreatment Prediction of Pathologic Complete Response to Neoadjuvant Chemotherapy in Breast Cancer: A Multicenter Study. CLIN CANCER RES2019, 25(12):3538-3547.

13. Parikh J, Selmi M, Charles-Edwards G, Glendenning J, Ganeshan B, Verma H, Mansi J, Harries M, Tutt $\mathrm{A}$, Goh V: Changes in primary breast cancer heterogeneity may augment midtreatment MR imaging assessment of response to neoadjuvant chemotherapy. RADIOLOGY 2014, 272(1):100-112.

14. Braman NM, Etesami M, Prasanna P, Dubchuk C, Gilmore H, Tiwari P, Plecha D, Madabhushi A: Intratumoral and peritumoral radiomics for the pretreatment prediction of pathological complete response to neoadjuvant chemotherapy based on breast DCE-MRI. BREAST CANCER RES 2017, 19(1):57.

15. Teruel JR, HeldahI MG, Goa PE, Pickles M, Lundgren S, Bathen TF, Gibbs P: Dynamic contrastenhanced MRI texture analysis for pretreatment prediction of clinical and pathological response to neoadjuvant chemotherapy in patients with locally advanced breast cancer. NMR BIOMED 2014, 27(8):887-896.

16. Thibault G, Tudorica A, Afzal A, Chui SY, Naik A, Troxell ML, Kemmer KA, Oh KY, Roy N, Jafarian N et al: DCE-MRI Texture Features for Early Prediction of Breast Cancer Therapy Response. Tomography 2017, 3(1):23-32.

17. Golden DI, Lipson JA, Telli ML, Ford JM, Rubin DL: Dynamic contrastenhanced MRI-based biomarkers of therapeutic response in triple-negative breast cancer. J Am Med Inform Assoc 2013, 20(6):1059-1066.

18. Michoux N, Van den Broeck S, Lacoste L, Fellah L, Galant C, Berliere M, Leconte I: Texture analysis on MR images helps predicting non-response to NAC in breast cancer. BMC CANCER 2015, 15:574.

19. Nilsen L, Fangberget A, Geier O, Olsen DR, Seierstad T: Diffusion-weighted magnetic resonance imaging for pretreatment prediction and monitoring of treatment response of patients with locally advanced breast cancer undergoing neoadjuvant chemotherapy. ACTA ONCOL 2010, 49(3):354-360. 
20. Chamming'S F, Ueno Y, Ferre R, Kao E, Jannot AS, Chong J, Omeroglu A, Mesurolle B, Reinhold C, Gallix B: Features from Computerized Texture Analysis of Breast Cancers at Pretreatment MR Imaging Are Associated with Response to Neoadjuvant Chemotherapy. RADIOLOGY 2018, 286(2):412-420.

21. Fan M, Wu G, Cheng H, Zhang J, Shao G, Li L: Radiomic analysis of DCE-MRI for prediction of response to neoadjuvant chemotherapy in breast cancer patients. EUR J RADIOL 2017, 94:140-147.

22. Minarikova L, Bogner W, Pinker K, Valkovic L, Zaric O, Bago-Horvath Z, Bartsch R, Helbich TH, Trattnig $\mathrm{S}$, Gruber S: Investigating the prediction value of multiparametric magnetic resonance imaging at $3 \mathrm{~T}$ in response to neoadjuvant chemotherapy in breast cancer. EUR RADIOL 2017, 27(5):1901-1911.

23. Liu Z, Wang S, Dong D, Wei J, Fang C, Zhou X, Sun K, Li L, Li B, Wang M et al: The Applications of Radiomics in Precision Diagnosis and Treatment of Oncology: Opportunities and Challenges. THERANOSTICS 2019, 9(5):1303-1322.

24. Gillies RJ, Kinahan PE, Hricak H: Radiomics: Images Are More than Pictures, They Are Data. RADIOLOGY 2016, 278(2):563-577.

25. Cain EH, Saha A, Harowicz MR, Marks JR, Marcom PK, Mazurowski MA: Multivariate machine learning models for prediction of pathologic response to neoadjuvant therapy in breast cancer using MRI features: a study using an independent validation set. Breast Cancer Res Treat 2019, 173(2):455463.

26. Xie T, Wang Z, Zhao Q, Bai Q, Zhou X, Gu Y, Peng W, Wang H: Machine Learning-Based Analysis of MR Multiparametric Radiomics for the Subtype Classification of Breast Cancer. FRONT ONCOL 2019, 9:505.

27. De Cecco CN, Ganeshan B, Ciolina M, Rengo M, Meinel FG, Musio D, De Felice F, Raffetto N, Tombolini V, Laghi A: Texture analysis as imaging biomarker of tumoral response to neoadjuvant chemoradiotherapy in rectal cancer patients studied with 3-T magnetic resonance. INVEST RADIOL 2015, 50(4):239-245.

28. Morrow M: Personalizing extent of breast cancer surgery according to molecular subtypes. BREAST 2013, 22 Suppl 2:S106-S109.

29. Pekar G, Hofmeyer S, Tabar L, Tarjan M, Chen TH, Yen AM, Chiu SY, Hellberg D, Gere M, Tot T: Multifocal breast cancer documented in large-format histology sections: long-term follow-up results by molecular phenotypes. CANCER-AM CANCER SOC 2013, 119(6):1132-1139.

30. Yang WT, Zhu XZ: [The introduction of $\mathbf{2 0 1 2}$ WHO classification of tumours of the breast]. Zhonghua Bing Li Xue Za Zhi 2013, 42(2):78-80.

31. Koh J, Park AY, Ko KH, Jung HK: Can enhancement types on preoperative MRI reflect prognostic factors and surgical outcomes in invasive breast cancer?EUR RADIOL 2019, 29(12):7000-7008.

32. Masuda N, Lee SJ, Ohtani S, Im YH, Lee ES, Yokota I, Kuroi K, Im SA, Park BW, Kim SB et al: Adjuvant Capecitabine for Breast Cancer after Preoperative Chemotherapy. N Engl J Med 2017, 376(22):21472159. 
33. Ford J, Dogan N, Young L, Yang F: Quantitative Radiomics: Impact of Pulse Sequence Parameter Selection on MRI-Based Textural Features of the Brain. Contrast Media Mol Imaging 2018, 2018:1729071.

34. Molina D, Perez-Beteta J, Martinez-Gonzalez A, Martino J, Velasquez C, Arana E, Perez-Garcia VM: Lack of robustness of textural measures obtained from 3D brain tumor MRIs impose a need for standardization. PLOS ONE 2017, 12(6): e178843.

35. Depeursinge A, Foncubierta-Rodriguez A, Van De Ville D, Muller $\mathrm{H}$ : Three-dimensional solid texture analysis in biomedical imaging: review and opportunities. MED IMAGE ANAL 2014, 18(1):176-196.

36. Cui Y, Yang X, Shi Z, Yang Z, Du X, Zhao Z, Cheng X: Radiomics analysis of multiparametric MRI for prediction of pathological complete response to neoadjuvant chemoradiotherapy in locally advanced rectal cancer. EUR RADIOL 2019, 29(3):1211-1220.

\section{Tables}


Table 1

Clinical and morphology characteristics between $\mathrm{pCR}$ and non-pCR group

\begin{tabular}{|llll|}
\hline Characteristics & pCR(n= 54) & Non-pCR(n= 73) & p value \\
\hline Age,mean(SD),Y & $52.80+9.24$ & $50.62 \pm 11.19$ & 0.309 \\
\hline Menonpausal status & & & 0.278 \\
\hline premenopaual & $15(27.78 \%)$ & $26(35.61 \%)$ & \\
\hline postmenopaal & $39(72.22 \%)$ & $47(64.39 \%)$ & \\
\hline Enhancement pattern & & & 0.299 \\
\hline Mass & $36(66.67 \%)$ & $53(72.60 \%)$ & \\
\hline non-mass & $18(33.33 \%)$ & $20(27.40 \%)$ & \\
\hline Multifocal or multicenter & & & \\
\hline present & $42(77.78 \%)$ & $53(72.60 \%)$ & \\
\hline absent & $12(22.22 \%)$ & $20(27.40 \%)$ & \\
\hline Pre-NAC T stage & & & 0.468 \\
\hline T2 & $40(74.07 \%)$ & $53(72.60 \%)$ & \\
\hline T3 & $14(25.93 \%)$ & $20(27.40 \%)$ & \\
\hline Pre-NAC N stage & & & \\
\hline N0 & $16(29.63 \%)$ & $23(31.50 \%)$ & \\
\hline N1 & $29(53.70 \%)$ & $38(52.05 \%)$ & \\
\hline N2 & $5(9.26 \%)$ & $2(2.74 \%)$ & \\
\hline N3 & $4(7.41 \%)$ & $10(13.71 \%)$ & \\
\hline Type of surgery & & & \\
\hline Conservation & & $9(12.33 \%)$ & \\
\hline Radical surgery & & $64(87.67 \%)$ & \\
\hline chemother & & \\
\hline
\end{tabular}

NAC, neoadjuvant chemotherapy PCR, pathological complete response SD, standard deviation 
Table 2

The accuracy, sensitivity, specificity and AUC of machine learning classifification based on optinal six features from $\mathrm{CE}_{1}$

\begin{tabular}{|lllll|}
\hline Classifier Model & accuracy & sensitivity & specificity & AUC \\
\hline Fine Tree & $59.1 \%$ & $48 \%$ & $67 \%$ & 0.61 \\
\hline Medium Tree & $59.1 \%$ & $48 \%$ & $67 \%$ & 0.62 \\
\hline Coarse tree & $60.6 \%$ & $31 \%$ & $82 \%$ & 0.61 \\
\hline Linear Discriminant & $68.5 \%$ & $52 \%$ & $81 \%$ & 0.69 \\
\hline Quadratic Discriminant & $66.1 \%$ & $43 \%$ & $84 \%$ & 0.66 \\
\hline Logistic Regression & $68.5 \%$ & $52 \%$ & $81 \%$ & 0.69 \\
\hline Linear SVM & $65.4 \%$ & $41 \%$ & $84 \%$ & 0.69 \\
\hline Quadratic SVM & $59.1 \%$ & $33 \%$ & $78 \%$ & 0.57 \\
\hline Cubic SVM & $49.6 \%$ & $48 \%$ & $51 \%$ & 0.49 \\
\hline Fine Gaussian SVM & $59.8 \%$ & $44 \%$ & $71 \%$ & 0.64 \\
\hline Medium Gaussian SVM & $62.2 \%$ & $35 \%$ & $82 \%$ & 0.70 \\
\hline Coarse Gaussian SVM & $66.1 \%$ & $29 \%$ & $95 \%$ & 0.68 \\
\hline Fine KNN & $60.6 \%$ & $54 \%$ & $66 \%$ & 0.60 \\
\hline Medium KNN & $59.8 \%$ & $44 \%$ & $71 \%$ & 0.67 \\
\hline Coarse KNN & $57.5 \%$ & $0 \%$ & $100 \%$ & 0.64 \\
\hline Cosine KNN & $63.8 \%$ & $59 \%$ & $67 \%$ & 0.62 \\
\hline Cubic KNN & $59.8 \%$ & $44 \%$ & $71 \%$ & 0.67 \\
\hline Weighted KNN & $60.6 \%$ & $52 \%$ & $67 \%$ & 0.64 \\
\hline Boosted Trees & $61.4 \%$ & $54 \%$ & $67 \%$ & 0.67 \\
\hline Bagged Trees & $59.1 \%$ & $52 \%$ & $64 \%$ & 0.65 \\
\hline Subspace Discriminant & $68.5 \%$ & $52 \%$ & $81 \%$ & 0.69 \\
\hline Subspace KNN & $60.6 \%$ & $54 \%$ & $66 \%$ & 0.60 \\
\hline RUS Boosted trees & $63.0 \%$ & $52 \%$ & $71 \%$ & 0.75 \\
\hline
\end{tabular}

KNN, k nearest neighbor SVM, support vector machine RUS, random undersampling ROC, receiver operating characteristic 
Table 3

The accuracy, sensitivity, specificity and AUC of machine learning classifification based on optinal six features from $\mathrm{CE}_{m}$

\begin{tabular}{|lllll|}
\hline Classifier Model & accuracy & sensitivity & specificity & AUC \\
\hline Fine Tree & $69.3 \%$ & $61 \%$ & $75 \%$ & 0.74 \\
\hline Medium Tree & $69.3 \%$ & $61 \%$ & $75 \%$ & 0.74 \\
\hline Coarse tree & $77.2 \%$ & $72 \%$ & $81 \%$ & 0.75 \\
\hline Linear Discriminant & $79.5 \%$ & $74 \%$ & $84 \%$ & 0.84 \\
\hline Quadratic Discriminant & $78.7 \%$ & $72 \%$ & $84 \%$ & 0.84 \\
\hline Logistic Regression & $79.5 \%$ & $74 \%$ & $84 \%$ & 0.84 \\
\hline Linear SVM & $79.5 \%$ & $74 \%$ & $84 \%$ & 0.84 \\
\hline Quadratic SVM & $70.1 \%$ & $61 \%$ & $77 \%$ & 0.74 \\
\hline Cubic SVM & $40.9 \%$ & $20 \%$ & $56 \%$ & 0.30 \\
\hline Fine Gaussian SVM & $74.8 \%$ & $69 \%$ & $79 \%$ & 0.77 \\
\hline Medium Gaussian SVM & $78.7 \%$ & $70 \%$ & $85 \%$ & 0.79 \\
\hline Coarse Gaussian SVM & $78.7 \%$ & $72 \%$ & $84 \%$ & 0.85 \\
\hline Fine KNN & $67.7 \%$ & $61 \%$ & $73 \%$ & 0.67 \\
\hline Medium KNN & $74.0 \%$ & $59 \%$ & $85 \%$ & 0.81 \\
\hline Coarse KNN & $57.5 \%$ & $0 \%$ & $100 \%$ & 0.78 \\
\hline Cosine KNN & $78.7 \%$ & $78 \%$ & $79 \%$ & 0.76 \\
\hline Cubic KNN & $74.0 \%$ & $59 \%$ & $85 \%$ & 0.81 \\
\hline Weighted KNN & $66.9 \%$ & $57 \%$ & $74 \%$ & 0.68 \\
\hline Boosted Trees & $67.7 \%$ & $61 \%$ & $73 \%$ & 0.76 \\
\hline Bagged Trees & $67.7 \%$ & $61 \%$ & $73 \%$ & 0.76 \\
\hline Subspace Discriminant & $79.5 \%$ & $74 \%$ & $84 \%$ & 0.84 \\
\hline Subspace KNN & $67.7 \%$ & $61 \%$ & $73 \%$ & 0.67 \\
\hline RUS Boosted trees & $68.5 \%$ & $65 \%$ & $71 \%$ & 0.75 \\
\hline
\end{tabular}

KNN, k nearest neighbor SVM, support vector machine RUS, random undersampling ROC, receiver operating characteristic 
Figures

advanced HER2 positive invasive breast cancer patients treated with NAT ( $\mathrm{n}=258)$

\begin{tabular}{|c|c|}
\hline & Excluded cases: \\
\hline & 1) Metastasis elsewhere in the body $(n=15)$ \\
\hline cluded cases & 2) No surgery was performed after NAT ( $n=13)$ \\
\hline & 3) premature discontinuation of $\mathrm{PCH}(\mathrm{n}=8)$ \\
\hline & 4) lack of baseline MRI or baseline MRI from other hospital ( $n=22)$ \\
\hline & 5) Occult breast cancer $(n=4)$ \\
\hline & poor MR image quality resulting from motion artefacts ( $\mathrm{n}=16$ ) \\
\hline
\end{tabular}

$\stackrel{\text { excluded cases }}{\longrightarrow}$ patients underwent MRI on other $\operatorname{scan}(\mathrm{n}=53)$

readmission cases

127 patients underwent MRI on $1.5 \mathrm{~T}$ scan (AURORA)

\section{Figure 1}

Flow chart of patient recruitment in this study 


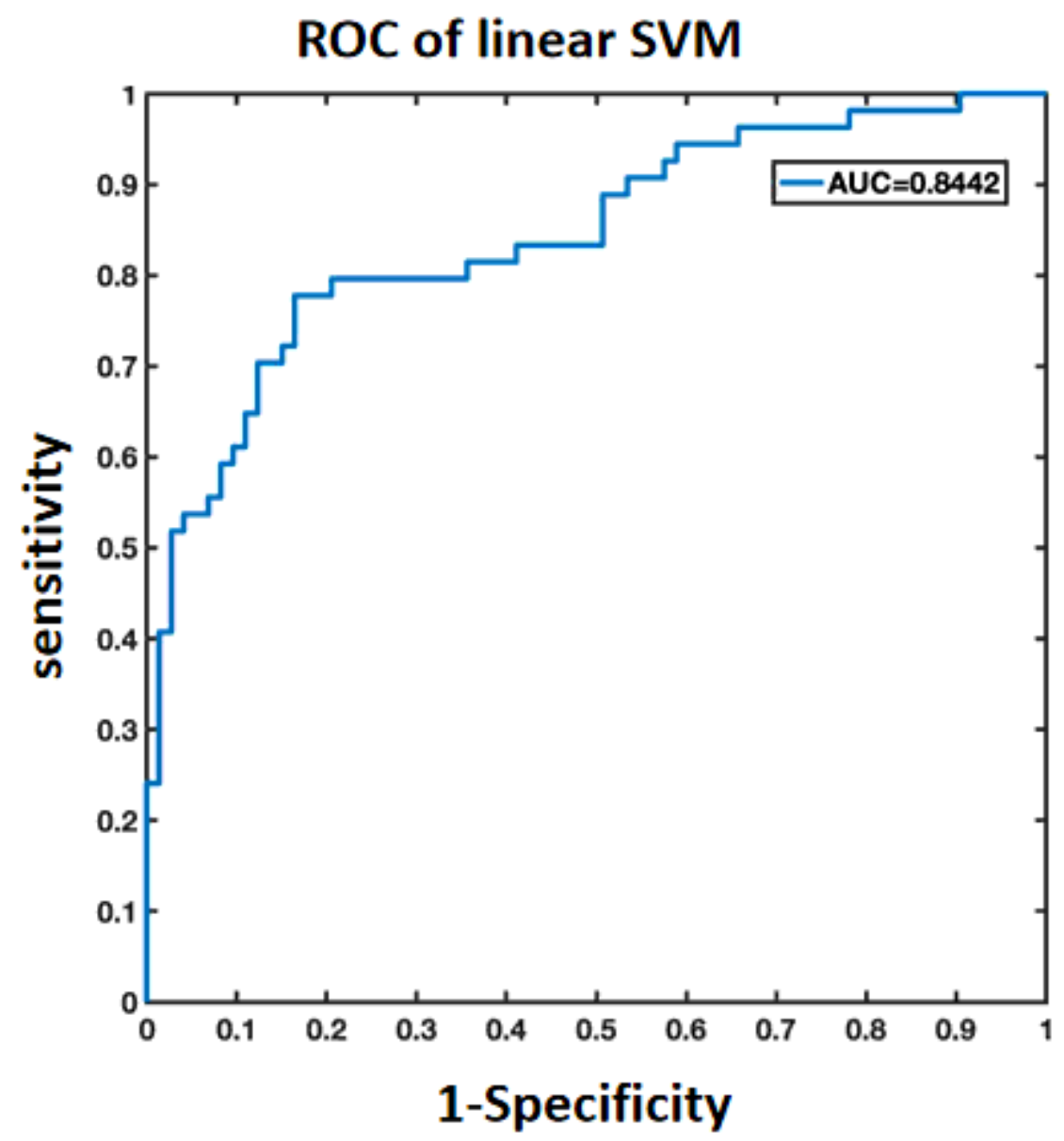

Figure 2

ROC of liner SVM predicting pCR and non-pCR 


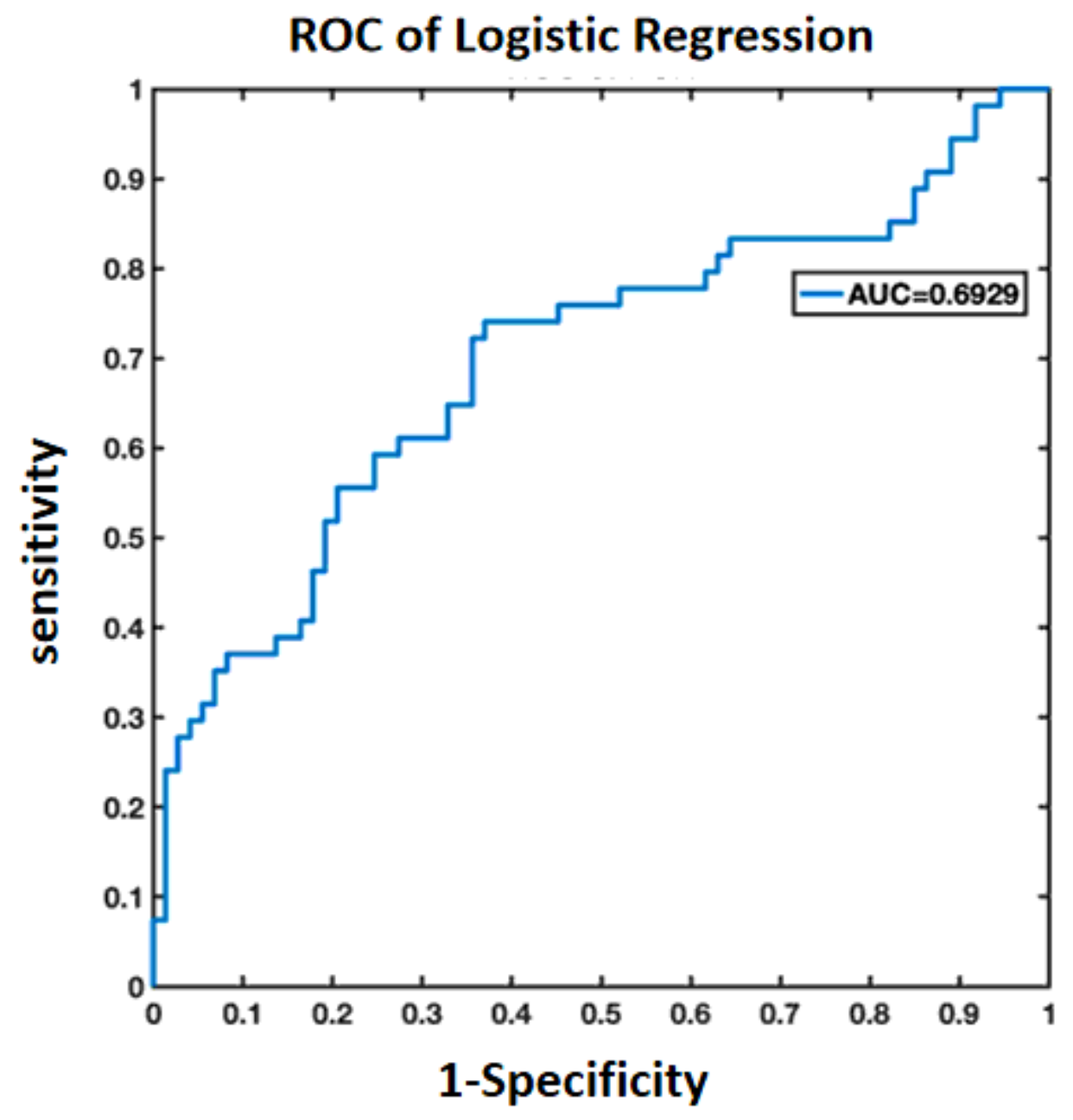

Figure 3

ROC of Logistic Regression predicting pCR and non-pCR 


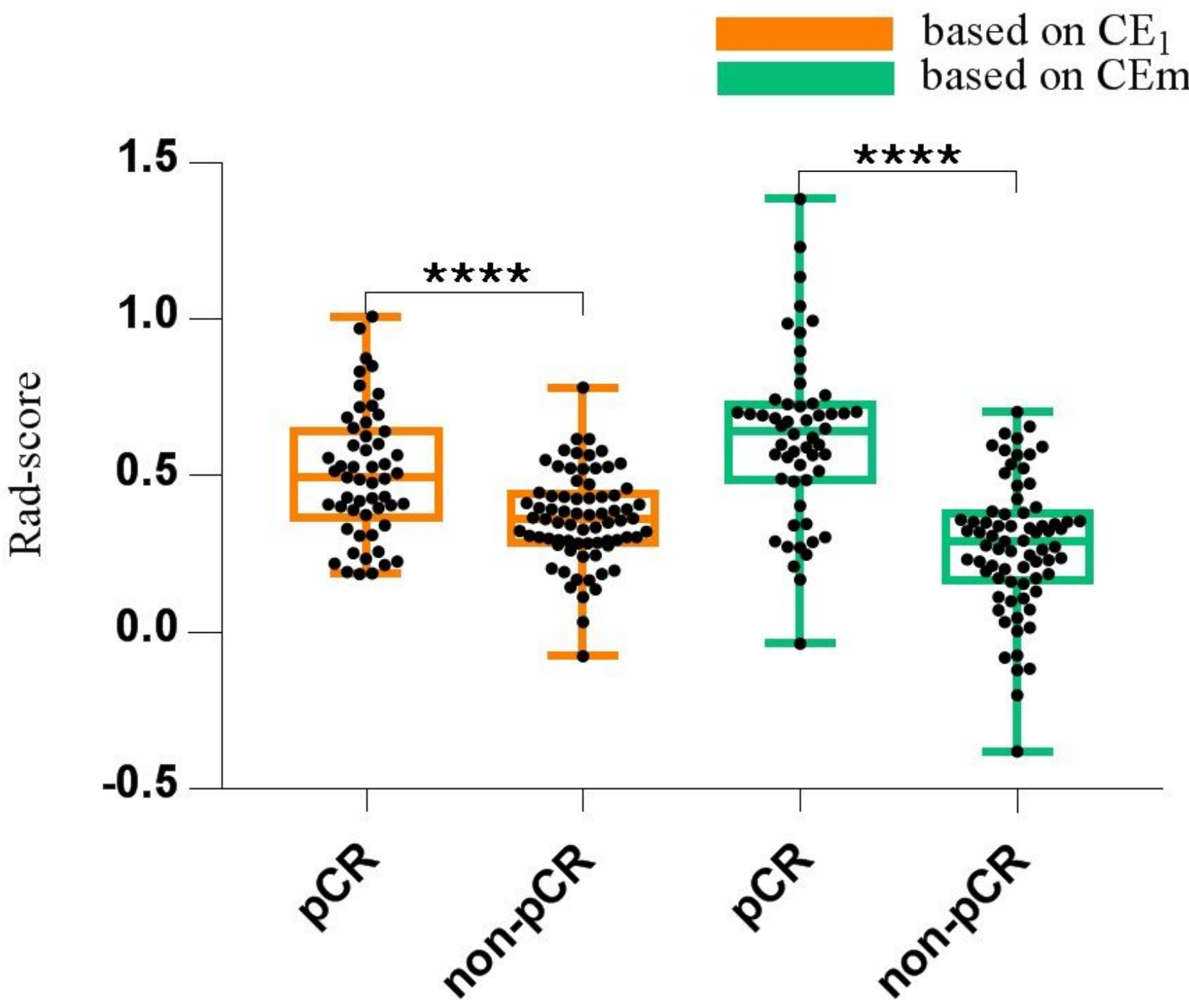

Figure 4

Rad-score box plot for pCR classification based on the CE1 and CEm

\section{Supplementary Files}

This is a list of supplementary files associated with this preprint. Click to download.

- SupplementaryMaterial.docx 